\title{
A Survey on Classical and Modern Face Recognition Techniques
}

\author{
M. ShalimaSulthana ${ }^{1}$, C. Naga Raju ${ }^{2}$ \\ ${ }^{1}$ Research Scholar, Department of Computer Science and Engineering, YSR Engineering College of YVU \\ Yogivemana University-Kadapa, Andhra Pradesh, India \\ ${ }^{2}$ Professor, Department of Computer Science and Engineering, YSR Engineering College of YVU, Yogivemana \\ University-Kadapa, Andhra Pradesh, India
}

\begin{abstract}
Article Info

Volume 7, Issue 6

Page Number: 57-79

Publication Issue :

November-December-2021

\section{Article History}

Accepted : 08 Nov 2021

Published : 19 Nov 2021

During the previous few centuries, facial recognition systems have become a popular research topic. On account of its extraordinary success and vast social applications; it has attracted significant study attention from a wide range of disciplines in the last five years - including "computer-vision", "artificialintelligence", and "machine-learning". As with most face recognition systems, the fundamental goal involves recognizing a person's identity by means of images, video, data streams, and context information. As a result of our research; we've outlined some of the most important applications, difficulties, and trends in scientific and social domains. This research, the primary goal is to summarize modern facial recognition algorithms and to gain a general perceptive of how these techniques act on diverse datasets. Aside from that, we also explore some significant problems like illumination variation, position, aging, occlusion, cosmetics, scale, and background are some of the primary challenges we examine. In addition to traditional face recognition approaches, the most recent research topics such as sparse models, deep learning, and fuzzy set theory are examined in depth. There's also a quick discussion of basic techniques, as well as a more in-depth. As a final point, this research explores the future of facial recognition technologies and their possible importance in the emerging digital society.
\end{abstract}

\section{INTRODUCTION}

The term "Face recognition research" has become extremely popular over the last few decades and it has a wide variety of scientific, social, and business applications. Face recognition has been major prominent research areas in "image processing", "machine learning", “computer vision", "artificial intelligence" and "visual surveillance" in the last five years. Face recognition systems must be able to determine a person's identity from static photos [1], video data [2] or data streams [3] in order to be effective.

A broad definition of face recognition encompasses a variety of technologies that can be used to create a facial recognition system. Image preprocessing and 
facial detection are some of the features. All faces in a picture are identified using a face identification method, which identifies the coordinate system of all the faces. That's the technique of scanning the entire image in order to identify if indeed the candidate area is a face or an object. Whereas if face recognition system is used, the outcome could be either square or rectangular. When a face is detected, its coordinates are stored in a face detection coordinate system. Basic positioning technologies are implemented in the deep learning framework.

Applications for face recognition technologies include attendance access control [4], finance and security [5]. In addition to logistics and retailing, Smartphone and mobile device use, transportation, real estate, education, government administration, activity promotion and information security of network [6]. When it comes to security, face recognition may be used to provide early warnings of potentially harmful circumstances, as well as to track down culprits of crime. Artificial intelligence has come a long way, so we need recognition technology that is more accurate, more adaptive, and faster than ever before.

Facial features as a human identity date back to the dawn of human civilization. Face recognition is superior than other conventional biometrics such as retina, iris or identification based on fingerprint since it can reveal reluctant persons in a timely manner. Though, face recognition is still a difficult and demanding topic when it comes to unconstrained environments, such as those with changeable illumination and various poses $[7,8]$, which have been the subject of many successful research results in recent years.

A person's face is the most often utilized biometric for identification and authentication. Face recognition based on Human Computer Interaction (HCI) uses either a feature-based or an image-based technique. Researchers have found that $[9,10,11]$ feature extraction based on facial recognition may be used in software development. Machine learning techniques like SVM and decision trees and Random-forest have improved facial recognition systems' performance by allowing them to recognize more faces with greater accuracy. In comparison to other approaches; the SVM classifier dramatically improved the recognition rate.

In our lifetime, we have learned to remember hundreds of faces and can recognize famous faces with a quick glance, even after years of separation. Global and local facial recognition algorithms are classified based on their features. In addition to global feature descriptor, it is also known as holistic system or holistic approach. A description is extracted from the face as a whole using these methods. As long as you take into consideration factors such as the viewing conditions and facial emotions as well as interruptions like hairdo or spectacles, the ability is fairly robust.

Since faces images are composite, multifaceted, and subject to vulnerable and liable to change over time [12], building a computer model for face identification and categorization is a difficult task. LBP is Simple but effective, the LBP identifies picture pixels by thresholding the adjacent ones. The resultant pixel is then regarded as binary [13]. An image's local structure is described by this nonparametric descriptor [14]. Only 3x3 pixels of an image can be utilized to build a mask with the LBP operator.

The benefit of LBP is its ability to adapt to variations in grey level luminance as well as its computational simplicity and ease of use. Although the basic LBP operator may capture dominating features in huge sale structures, its small 3X3 mask neighborhood limits its ability to do so. Face analysis can benefit from the newly introduced LBP features; they were originally designed to extract features. When it comes to expression and pose, LBP is very resilient. Gray- 
level disparities generated by light discrepancies are insensitive to it as well. As a result of LBP's robustness, a variety of LBP-based face recognition systems have been created. [15].

As a result of its wide range of applications, face identification and analysis has been a prominent focus of computer vision research in recent decades. "Fisher face" [16], "Gabor feature" [17], "Scale-Invariant Feature Transform features" [18] (SIFT) , "Principal Component Analysis" [19] (PCA), "Sparse Depictedbased Classification algorithm" [20], "Nearest Features Line-based Subspace Analysis" [21], "Neural Networks" (NN) [22], "Wavelets" [23], "Independent Component Analysis" [24] (ICA), and "Kepler's algorithm" [25] are some of the notable contributions The performances and robustness of face recognition system have been improved dramatically. Changes in the surroundings, such as illumination, emotion variance, and pose alterations have an impact on the output of several procedures. Finding a technique that can handle these variations is difficult. Recognition and classification of facial expressions can be used to improve communication between man and machine. Another key difficulty for real-world facial emotional credit systems [26] is to make face identification more dependable under unrestricted lighting situations.

\section{Face recognition techniques :}

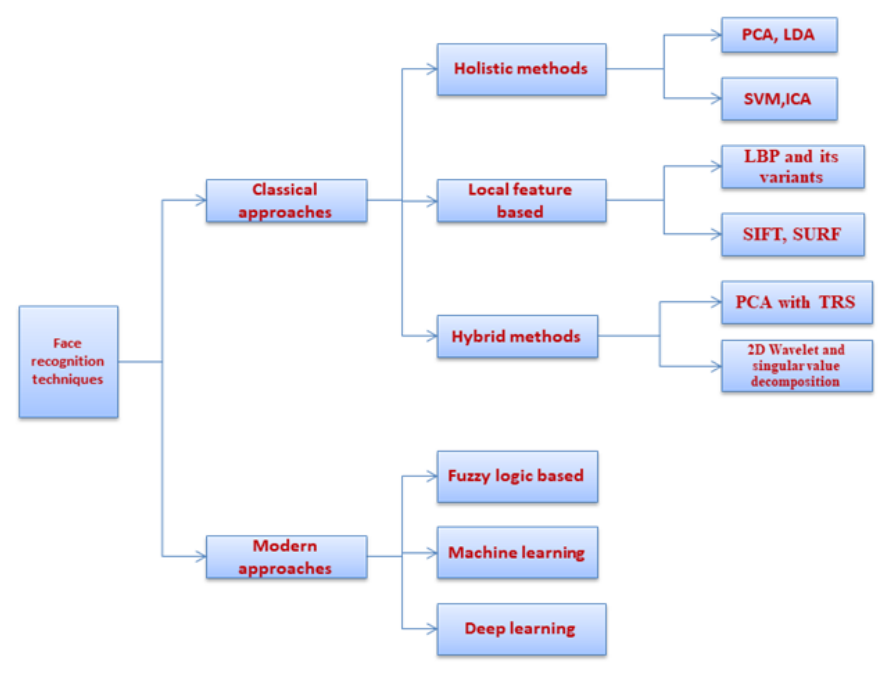

Fig1: shows various face recognition techniques

\section{Classical Approaches:}

As far back as the 1950s in "psychology" and the 1960 s in "engineering literature", face recognition research has lengthy history. According to Lawrence Gilman's (MIT), these concepts were derived from pattern recognition systems. He discovered that twodimensional features derived from a photograph can be matched to a three-dimensional representation. From following investigation, it was discovered that practical issues in varying climatic circumstances still exist, even with today's sophisticated supercomputers and GPUs. Despite the fact that these early research approaches were based on geometrical correlations between facial locations, they were driven by pattern recognition.

The majority of these methods rely heavily on the recognition of these facial features in difficult locations, as well as the constancy of these correlations across numerous variations. For the research community, these challenges remain a significant burden. Ref [27] made another early attempt at face recognition, Total 16 facial attributes are extracted using simple image processing algorithms. It achieved a 75 percent accuracy rate on a specified 20 people database of utilizing two photos 
per person using a basic Euclidean distance calculation.

There are three different types of face recognition techniques: holistic methods (i.e. PCA and LDA, SVM, ICA,) as well as features-based methods such as the "dynamic link architecture and the Hidden Markov Model and Convolution Neural Network (Modular Eigen faces, Hybrid LFA, Shape normalized, and component based methods)".

\section{1) PRINCIPAL COMPONENT ANALYSIS:}

PCA is an extracted feature approach designed for enhancement and application in this study. Due to the intensity of (PCA), which sets the stage for extracting a characteristic based on the evaluation of all data sets, this strategy should look into the dataset, which suggests that the image will not be reviewed and its attributes will not be extracted separately. As a result, not only must the empirical dataset be chosen correctly for the training phase, but also to eliminate the function required evaluating the dataset. It also suggests that the characteristic for each examine image cannot be extracted separately; the results must be compared, and the PCA algorithm must be used.

PCA is a computational empirical method that is mostly employed in picture recognition, according to various findings in the literature.PCA is one of the most common approaches to improve a data element while preserving as much information as possible. This approach is applied in a variety of ways, such as mathematical models, computational biology, image analysis, and data processing, to name a few.

Auto faces are a line arrangement of weighted eigenvectors used in the PCA method to represent faces. The image database is used to create these eigenvectors. Matrices of covariance the quantity of photographs in the database will determine the proportion of own faces obtained. For multidimensional hyperspace data analysis, PCA is a suitable tool. Data extraction is an unsupervised learning technique that assumes data structure information is not there.

The most extensively used data dimensionality reduction approach is Principle Component Analysis (PCA). Face extraction is performed in facial recognition using an algorithm that aids PCA. MIT Media Lab's Turk and Pentland were introduced in 1991. Facial recognition relies heavily on analysis [34]. PCA is commonly used to convert data from one format to another. More angles are used to analyze facial data. Remove unneeded information and noise while keeping the essentials. Data characteristics significantly minimize measurements, speed up data processing, and save time and money [35] [36]. As a result, this algorithm is widely employed. View involving dimensional reduction and multidimensional data.

Using Eigen Values the original data is projected into a feature space with a lesser dimension, defined by Eigen vectors, using PCA covariance matrix can be produced. The Eigenvectors calculated by PCA are called Eigen faces and have been employed in face representation and recognition. More than human faces, gel images have a high dimensionality compared to their size, making PCA a helpful first step in analysis. Many methods exist for recognizing faces, including, PCA principle component analysis known as Eigen faces is a method for analyzing and matching features. To track human movement, a combination of feature matching and feature selection was used in the past to track the faces of people using Eigen faces.

\section{Steps followed by PCA are:}

Step1: Assume $\mathrm{K}$ images $\left(\mathrm{I}_{1}, \mathrm{I}_{2}, \mathrm{I}_{3} \ldots \mathrm{I}_{\mathrm{k}}\right)$ by $\mathrm{X}^{*} \mathrm{X}$ size having $\mathrm{X}^{2}$ row or column size of vector.

Step2: the training set image average $(\mu)$ is calculated by

$\mu=\frac{1}{K} \sum_{x=1}^{K} I x$ 
Step3: By vector $(\Phi)$, from the average image each trainee image is differs.

$\Phi_{\mathrm{i}=\mathrm{I}^{-}-\mu}$

Step4: the Covariance Matrix or Total Scatter Matrix , is calculated as follows:

Cove $=\frac{1}{K} \sum_{x=1}^{K} \Phi \mathrm{n} \Phi_{\mathrm{n}}{ }^{\mathrm{T}}==$ AAT, where $\mathrm{A}=[\Phi 1 \Phi 2 \Phi 3 \ldots$ $\Phi \mathrm{n}]$

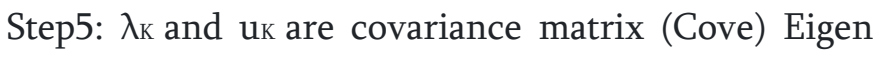
values and eigenvectors

Step6: Calculate the vectors of weights, to image classification; which can be projected into this feature space.

$\Omega \mathrm{T}=\left[\omega 1, \quad \omega 2 \ldots \quad \omega \mathrm{M}^{\prime}\right], \quad$ Where $\quad \omega \mathrm{k}=\mathrm{uk}^{\mathrm{T}}(\mathrm{k}-\mu)$ $\mathrm{k}=1,2, \ldots \ldots \ldots \ldots \mathrm{M}^{\prime} \quad$ (4)

here M' represents not only total Eigen faces, but the ones with greater values.

In Eigen face, one of the classical approaches is a PCA-based feature extraction algorithm [37]. The simplest method of feature extraction is shown in Figure 2, in which PCA faces are joined. The K-NearNeighborhood (KNN) technique was used to verify the results. Get covariance eigenvectors by selecting a matrix and major component from a set of sample data and computing Eigen values and eigenvectors of covariance.

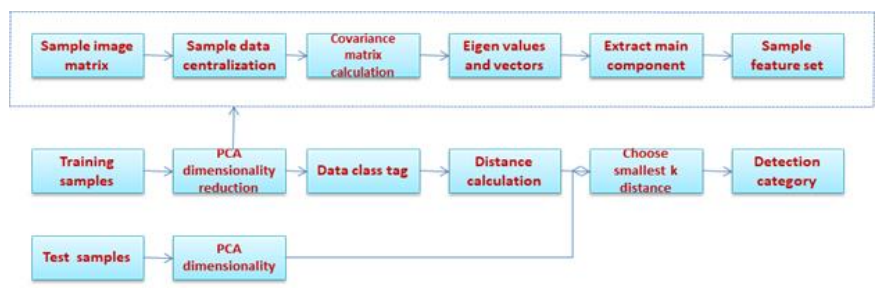

Fig 2: Principle Component Analysis with KNN

Technique used to verify the result

Despite the fact that PCA is effective at managing big data sets [38]. The most significant disadvantage is that your training dataset must be sufficiently large [39]. as if there are thousands of original images in the facial recognition system, then its difficult to collect Low-dimensional data as if persons have a wide range of facial expressions, and there are obstructions in front of the face, and the light is excessively strong or weak.

\section{PCA limitations:}

Consequently, PCA is vulnerable to change in illumination since it treats the inner-class and outerclass equally.

\section{2) LDA:}

LDA linear discriminant analysis is a dimensionality reduction technique used for supervised classification problems. It's utilized to separate two or more classes by modeling differences in groupings. Using these technique Higher-dimensional characteristics are transformed into a lower-dimensional environment. LDA is a "classic" technique for determining a linear combination of characteristics that characterizes or distinguishes numerous classes of objects, as well as reducing dimensionality before classification.

An individual face is represented by an array of pixel values in computerized facial recognition. Specifically, to minimize the total number of features before classification, linear discriminant analysis is used. Fisher's linear discriminant and Eigen faces are the terms used to describe the linear combinations obtained using principle component.

Every new dimension is then constructed from Fisher linear combinations, which is the term given to the linear combinations derived by utilizing Fisher's Linear Discriminant (Fisher).while Eigen faces are the name given to the linear combinations obtained using principle component.

\section{Class-dependent transformation:}

This strategy focuses on increasing the between-class to within-class ratio, major goal is maximizing the ratio in order to provide acceptable class separation. If you're going to use a class-specific type strategy, for transforming the data sets independently you'll need to use two optimizing criteria's.

\section{Class Independent transformation:}

Using this technique the ratio of total variation to within-class variance is maximized. Since only one optimization principle is used to convert the data sets, this strategy transforms every data point, regardless of their class identity. When implementing this kind of LDA, each class is treated as single compared to all 
other classes. A lower-dimensional projection of the original data matrix is achieved using LDA. This required three stages. Separability between classes should be determined as a first step. In the second step, compute the within-class variance (within-class matrix) in order to determine how much difference exists between a class mean and its representative samples. In the third step, build a lower-dimensional space that maximizes variance across classes while minimizing variance within classes.

We can apply linear discriminatory analysis (LDA) to analyze the labeled facial recognition dataset [40]. It's used to talk about classification [41].As shown in Fig. 3 , After dimensionality reduction, PCA requires as much data variance as possible in order to partition the data as broadly as possible, whereas LDA demands as little variance within the same data group category as possible after projection and as much variance across groups as possible [42]. This indicates that LDA has taken care of reducing dimensionality, and you should use the information on the label as much as possible to distinguish the various kinds of data.

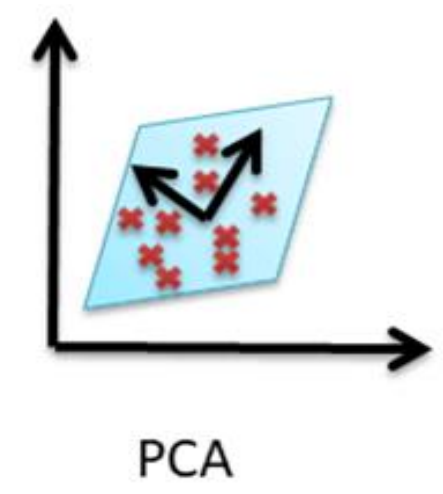

PCA: Component axis that Maximize the variance

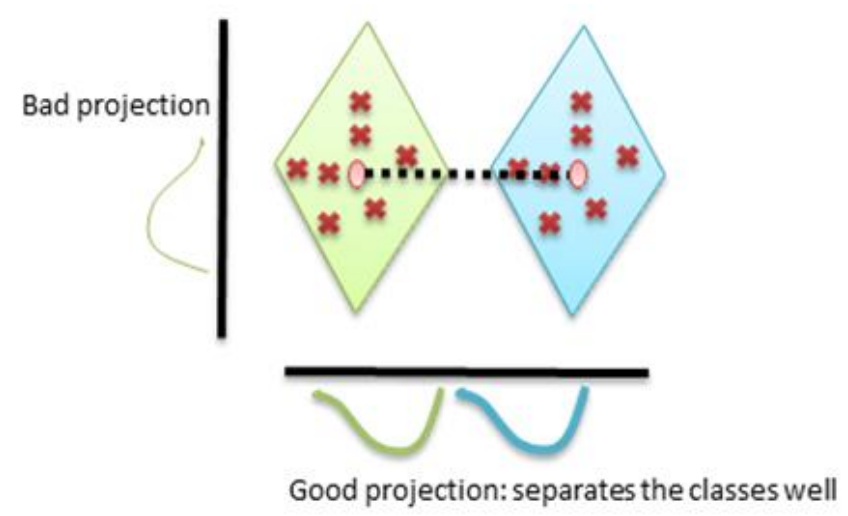

LDA: Maximizing the component Axis for class separation

Fig 3: shows comparison between PCA and LDA

Fig 3: shows while principle components analysis (PCA) focuses on the data as a whole rather than focusing on the actual class structure. Unlike PCA, LDA does not alter the original data sets' positions, but instead aims to improve class separability by drawing a decision region between the specified classes. When employing Linear Discriminant Analysis, face vector space must be represented efficiently (LDA), by combining all of the data from facial training, PCA creates a face space that does not rely on face classes. Class-specific information is used in LDA. Which maps data into a classification space by generating linear discriminant function, where the sample's categorization is determined using metrics like Euclidean distance. As a result of LDA, an object's numerous properties are analyzed to determine which group it is most likely a part of.

\section{3) Independent Component Analysis ICA:}

Most successful face recognition representations that use PCA include Eigen faces [43], holons [44], and local feature analysis [45]. Because much of the crucial data in a task This means it is vital to test whether or not PCA generalization that takes into account high-order correlation between picture pixels, rather than only second-order associations, is beneficial for face recognition. An example of this is Independent Component Analysis (ICA) [46]. 
ICA representations were benchmarked against PCA, which is similar to the "Eigen faces" representation [47], [48].

[49] [50], and [51] are some of the algorithms for performing ICA. Information is optimally transferred in neurons with sigmoid transfer functions [52], hence we used Bell and Sejnowski's infomax technique.

This algorithm works as follows:

Let $\mathrm{Y}$ is $\mathrm{n}$-Dim random vector represents inputs distribution and $Z$ represents $n x n$ invertible matrix, $\mathrm{V}=\mathrm{YZ}$ and $\mathrm{X}=\mathrm{f}(\mathrm{V})$ an $\mathrm{n}-\mathrm{Dim}$ random variable represents the $n$-neurons outputs.

Invertible squashing functions are used to map real numbers into the interval $[0,1]$ in each component of $\mathrm{f}=(\mathrm{f} 1$ fn).

$$
\mathrm{f}_{\mathrm{i}}(\mathrm{v})=\frac{1}{1+e}-\mathrm{u}
$$

Here $\mathrm{V}_{1}$ $\ldots V_{n}$ are linear variables can be regarded as pre synaptic activations of -neurons

$\mathrm{Y}_{1} \ldots \ldots \ldots . . . . . \mathrm{Y}_{\mathrm{n}}$ are post synaptic activations delimited by the $[0,1]$ interval.

The weight matrix's gradient updating rule is as follows WM:

$\Delta \mathrm{WM} \propto \nabla_{\mathrm{Wm}} \operatorname{En}(\mathrm{Q})\left(\mathrm{W}^{\mathrm{T}}\right)^{-1}+\mathrm{V}\left(\mathrm{Q}^{\prime} \mathrm{P}^{\mathrm{T}}\right)$

$\mathrm{W}^{\mathrm{T}}=$ Transpose of weight matrix; V=Expected Value; $\operatorname{En}(\mathrm{Q})=$ random vector $(\mathrm{Q})$ entropy

$\nabla_{\mathrm{wm}} \operatorname{En}(\mathrm{Q})=$ Matrix form representation of gradient of entropy

Natural gradient (1) is calculated by multiplying the absolute gradient by WTW , this can be used to avoid computing the matrix inverse.

$\Delta \mathrm{WM} \alpha \nabla_{\mathrm{WM}} \mathrm{En}(\mathrm{Q}) \mathrm{W}^{\mathrm{T}} \mathrm{W}=\mathrm{I}+\left(\mathrm{Q}^{\prime} \mathrm{V}^{\mathrm{T}}\right) \mathrm{WM}$

I represents identity matrix; $\mathrm{Q}^{\prime}=\left(1-2 \mathrm{Q}_{\mathrm{i}}\right)$

When there are several inputs and an output, increasing the output's joint entropy encourages the separate outputs to become statistically independent.

The following are some of the possible benefits of ICA over PCA: 1) it gives a more accurate statistical data model, allowing for a more accurate recognition of data concentrations in $\mathrm{n}$-dim space. 2) The mixing matrix is uniquely identified by LDA. 3) It may be able to reconstruct data more accurately than PCA in the presence of noise. 4) It is sensitive to high-order statistics in data, in addition to the covariance matrix.

\section{4) Support vector machine (SVM):}

SVM was projected by "Vapnik and Cortes" in 1995. SVM [53] is a facial recognition technique designed for short samples and high-dimensional challenges. A generalized portrait algorithm was used to create this classifier. It quickly became the most popular machine learning technology due to its superior text classification performance [54]. We employ facial features extracted and SVM to find the hyper plane to discriminate various faces in facial recognition. Assume you have a 2dim space with a lot of training data. To successfully categorize training data, SVM must discover a collection of straight line. The supporting vector is the data point, represented in Fig.4; this segmentation technique has the greatest generalizability. The approach described above separates data in a 2Dim plane; however the principle can be used to $3 \mathrm{dim}$ or even higher-dimensional space, with the limitation that the discovered boundary becomes a plane or hyper plane.

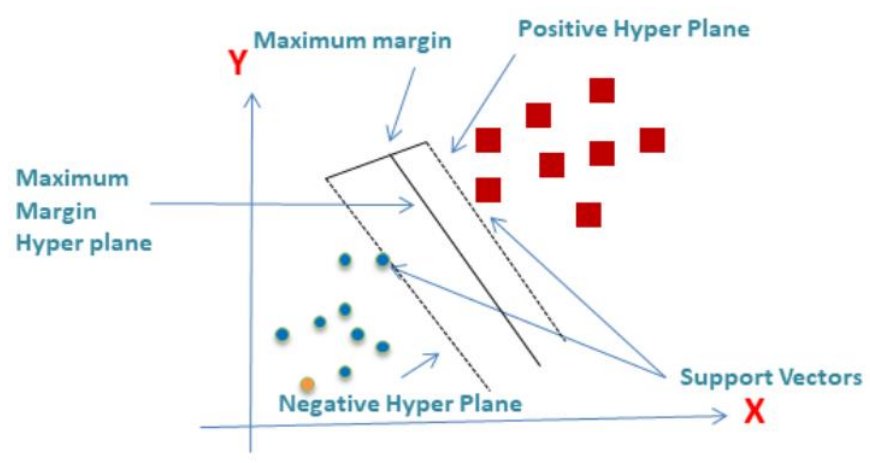

Fig 4 : support vector machine representation

\section{SVM works as follows:}

Support Vector Machine is a binary classification approach based on the concept of structural risk 
reduction that finds the best linear decision surface. The decision surface is a weighted mixture of the training set's features. The boundary between the two classes is defined by these items, which are called support vectors.

A set of labeled training data $\left(\mathrm{Mi}, \mathrm{N}_{\mathrm{i}}\right)$ is fed into an SVM algorithm, where $\mathrm{M}_{\mathrm{i}}$ is the data and the label $\mathrm{Ni}$ $=-1$ or 1 . A SVM approach produces a collection of support vectors $\mathrm{Svi}$, and $\mathrm{Ci}$ coefficients weights, support vector class labels $\mathrm{N}_{\mathrm{i}}$, and a constant term $\mathrm{b}$. The decision surface is linear calculated as.

$\mathrm{w} . \mathrm{z}+\mathrm{b}=0$

$\mathrm{W}=\sum_{i=1}^{\mathrm{Vs}} \mathrm{ci} \mathrm{N} \mathrm{S}_{\mathrm{i}}$

By implementing a kernel Ker (".) SVM may be extended to nonlinear decision surfaces. The decision surface is nonlinear.

$\sum_{i=1}^{\mathrm{Vs}} \mathrm{ci} \mathrm{N} \operatorname{Ker}\left(\mathrm{Svi}_{\mathrm{i}} \mathrm{z}\right)+\mathrm{b}=0$

An image of a person's face vector represented by $\mathrm{P} \quad \mathrm{T}$ $\mathrm{R}^{\mathrm{n}}$, where face space represented by $\mathrm{R}^{\mathrm{n}}$. Face space might be the original vectorized pixel values or another feature space, such as projecting the facial image on the eigenvectors created by applying PCA on a training set of faces (also referred to as Eigen faces).

If $\mathrm{P}_{1}$ and $\mathrm{P}_{2}$ are photos of the same face, we write $\mathrm{P}_{1}=$ $\mathrm{P}_{2}$ and $\mathrm{P} 1$ \# $\mathrm{P}$ if two images are different. To minimize ambiguities, we used the following nomenclature for identification and verification. Where as an unknown face is represented by the probe, while the gallery is a collection of photographs of recognized persons. The face in identification a probe has been identified. A probe is the facial image displayed to the system during verification. Whose identification must be confirmed the probe set refers to the collection of unknown faces.

SVM was created to classify binary data. Face recognition is a multi-class classification problem.
Two types of SVM algorithms are used to recognise faces: One versus One and One against All. As a result of this technique, each pair of classes is categorized. In the "one against all" category, each class is separated from the other classes.

We frequently have to deal with $\mathrm{n}>=2$ classes in realworld scenarios. Two pairs make up the training set

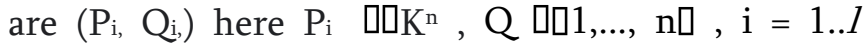
extending the two-class to the multiclass case for. $\mathrm{N}$ SVMs are trained in the one-vs-all technique. Each SVM distinguishes a all other classes from single class.

Each of the $\mathrm{n}$ individuals in the face database has ten face images. The training samples were made up of five photos from each of the ten, while the test samples were made up of the remaining five. The other training samples' photographs were all classified as negative samples, but the first participant's five shots were designated as positive samples. In order to generate acceptable support vectors and the optimal hyper plane, positive and negative data were used as input samples to train an SVM classifier. The SVM was given the name SVM1. As a result, we can calculate the SVM for each individual, which is denoted by the letters $\mathrm{SVM}_{1} \ldots \mathrm{SVM}_{\mathrm{n}}$.

The samples can be divided into $\mathrm{n}$ classes using the $\mathrm{n}$ SVMs. There would be various scenarios if a test sample was fed into each SVM in turn:

The sample was classed as class I if $S_{V M}$ determined it to be positive while other SVMs determined it to be negative at the same time.

$>$ If multiple SVMs decided to be negative at the same time while other SVMs decided to be positive, the classification was erroneous.

$>$ If all SVMs simultaneously decided that the sample was negative, it was determined that the sample did not belong in the face database. 
SVMs were utilized for classification with a variety of feature extraction methods: PCA, LDA, and ICA are three different types of investigation.

\section{Local Featured Based Methods:}

Based on the input image's local features, local feature-based algorithms can identify unique face regions like mouth, eyes or the nose and then calculate the geometric relationships between those facial spots. Ref [55] presented the algorithm that is most commonly used, Local Binary Pattern. Before collecting histograms of local binary pattern, first the facial image is segmented into small regions of interest. The feature classification is done using the closest neighbor classifier. It was a groundbreaking concept at the time, and it's still used in most facial recognition systems today.

Ref [56] proposes a Descriptor for generalized LBP texture that makes effective use of local texture features. For changing the illumination effect, the approach is quite easy and efficient while keeping the fundamental appearance elements required for recognition. LBP-based recognition is also greatly improved by removing the local histogram and substituting it with similarity measures based on local distance transformed.

When it comes to parameter selection, the LBP operator is extremely powerful, and while minor changes in the parameter can result in significant variations in feature vector size, A $3 \times 3$ sample size's LBP is validated as

$$
\begin{aligned}
& L B P=\sum_{i=0}^{7} S(\mathrm{~Pa}-\mathrm{Pb}) 2^{\mathrm{i}} \\
& \mathrm{S}(\mathrm{x})=\left[\begin{array}{c}
1 \text { if } \mathrm{x}>=0 \\
0 \text { if } \mathrm{x}<0
\end{array}\right.
\end{aligned}
$$

Where $\mathrm{Pa}$ denotes the central pixel and $\mathrm{Pb}$ denotes neighboring pixels. The binary code is created by associating a value to its surroundings. It's not affected by changes in lighting or contrast. Small fluctuations in pixel values and noise, however, make it vulnerable.

\section{UNIFORM LBP:}

LBP operator has been replaced by a uniform LBP operator (ULBP). Use rotation-invariant facial features to decrease the feature vector. When the bit pattern is circularly at right angles to each other, there are only two in-between transforms from 1 to 0 or vice versa. $(8, \mathrm{R})$ neighborhood will produce 59 patterns from an array of bits of 256. It is possible to calculate the pattern by using $2 \mathrm{p}$. $\mathrm{ULBP}=\left|\mathrm{M}\left(\mathrm{Pa}_{-1}-\mathrm{P}_{\mathrm{b}}\right)-\left(\mathrm{P}_{0}-\mathrm{P}_{\mathrm{b}}\right)\right|+\mid \quad \sum_{a=1}^{a-1} \mathrm{Q}(\mathrm{Pa}-$ $\mathrm{Pb})-\mathrm{Q}(\mathrm{Pa}-1-\mathrm{Pb})$

The rotation invariance of LBP is used to map the uniform pattern, with the goal of obtaining fundamental aspects of face images.

\section{SIFT:}

SIFT method proposed by David G Lowe in 2001 [57]. SIFT stands for Feature-Based Face Recognition. SIFT aids in the extraction of local features from photos. SIFT's implementation technique is divided into five stages: 1 .Scale space extreme detection, which detects image key points; 2.Getting Rid of Unreliable Key points 3.Keypoints direction, where the gradient of magnitude to those key points is determined 4.Feature descriptors are the results of the calculated feature of key points. 5. Comparing and contrasting the aspects of two separate photographs. Initially SIFT Algorithm performs feature extraction on test images. These features are compared to SIFT features as a result of the training image .Based on Euclidian distance, nearest neighbor matching is used to match the two features.

Step 1: Extreme Detection of Scale-Space: To begin, we use SIFT to discover a few key points on the face. The Difference-Of-Gaussian function is used to derive the image's scale space. By examining the image scale space, you may find the maxima and minima key points. Convolution of the Gaussian function GF ( $\mathrm{x}$, $\left.\mathrm{y}^{\mathrm{i}}, \sigma\right)$ with an input image I generates the image's scale space $\left(\mathrm{xi}_{\mathrm{i}}, \mathrm{y}_{\mathrm{i}}\right)$.

$$
\mathrm{L}(\mathrm{xi}, \mathrm{yi}, \sigma)=\mathrm{GF}\left(\mathrm{xi}, \mathrm{yi}_{\mathrm{i}} \sigma\right)^{*} \mathrm{I}\left(\mathrm{xi}, \mathrm{yi}_{\mathrm{i}}\right)
$$


Where GF ( $\left.\mathrm{xi}, \mathrm{yi}_{\mathrm{i}}, \sigma\right)$ is the Gaussian convolution kernel. The pixel coordinates of the input image are represented by $\left(\mathrm{x}_{\mathrm{i}}, \mathrm{y}_{\mathrm{i}}\right)$.Scale factor is represented by $\sigma$.The scale space images are $\mathrm{L}(\mathrm{xi}, \mathrm{yi}, \sigma)$.

The multiplicative factor $\mathrm{k}$ is used to discriminate nearby scales of two independent Gaussian pictures, yielding Local Maxima or Minima of $\mathrm{F}_{1}\left(\mathrm{x}_{\mathrm{i}}, \mathrm{y}_{\mathrm{i}}, \sigma\right)$

$$
\begin{aligned}
\mathrm{F}_{1}\left(\mathrm{x}_{\mathrm{i}}, \mathrm{yi}_{\mathrm{i}} \sigma\right) & =\left(\mathrm{GF}\left(\mathrm{x}_{\mathrm{i}}, \mathrm{yi}_{\mathrm{i}} \mathrm{k} \sigma\right)-\mathrm{GF}\left(\mathrm{x}_{\mathrm{i}}, \mathrm{y}_{\mathrm{i}}, \sigma\right)\right){ }^{*} \mathrm{I}\left(\mathrm{x}_{\mathrm{i}}, \mathrm{y}_{\mathrm{i}}\right) \\
& =\mathrm{L}\left(\mathrm{x}_{\mathrm{i}}, \mathrm{yi}_{\mathrm{i}}, \mathrm{k} \sigma\right)-\mathrm{L}\left(\mathrm{x}_{\mathrm{i}}, \mathrm{y}_{\mathrm{i}}, \sigma\right)
\end{aligned}
$$

For each surrounding scale in which the sample point and its eight neighbors' have been measured against each other, $\mathrm{F}(\mathrm{x}, \mathrm{y}, \sigma)$ 's maximum and minimum values are calculated. As a candidate key point, only pixels that reflect a local maximum or minimum among all the compared pixels can be evaluated.

Step 2: Unpredictable Key point's elimination: Final key points are chosen based on their stability measurements. Because there are so many key points while creating extreme key points, this phase assists in removing the unreliable and unstable key points. During this process, some key points that are susceptible to noise or have no edge effect are removed. The crucial point is removed if the value goes below a specified level.

Step 3: Key point Direction: The direction of the key point, which can offer feature rotation invariance, is the following step.

Step 4: Key point feature Descriptor: A 16*16 key point neighborhood descriptor is constructed by calculating orientation and the gradient magnitude at each picture point. An orientation histogram of size $4 * 4$ has been created by using the Gaussian window to weight the neighborhood and collect this weight. Histograms consist of 8 categories; hence each feature has $4 \mathrm{X} 4 \mathrm{X} 8=128$ key point descriptors.

Step 5: Matching Feature: This step is same as image matching. It's all about matching features in an image. On each of the two images, Euclidian distance is used to match two key points. The key point descriptor produced from the test image is then compared to the key point descriptor extracted from the training images. For each key point descriptor, the best match is obtained by selecting the nearest neighbour in the database of key point descriptors from the training pictures. A second threshold is utilized to eliminate the key points that do not have a good enough match with each other. There are two factors that determine whether to accept or reject a match: nearest neighbor and second-closest neighbor's distance.

\section{SURF (Speeded Up Robust Features):}

It's called SURF, Bay and his colleagues proposed the SURF algorithm. While SURF's performance is equivalent to that of SIFT, it reduces the computational complexity [58], Surf finds image key points and produces descriptive text. A Hessian matrix is used to determine the key points in SURF's algorithm. Applying appropriate filters to the integral image simplifies the operation and reduces the computing cost. Calculate Haar wavelet responses in both directions to find out what's going on. A robust key point descriptor is discovered by SURF technique employing integral picture and Hessian matrix. Key points from two facial photos are then compared for recognition.

In the SURF algorithm feature points speak about the points that appear in specific locations in images, such as edge points, corner points and spot points. To determine key points reliability repeatability used as an important performance metric. The Hessian matrix is utilized in the SURF technique to speed up the process. The Hessian Matrix can be used to identify the maximum value point.

In image I, scale $\Sigma$ at the Hessian matrix at a position $\mathrm{X}=(\mathrm{x}, \mathrm{y})$ in image ' $\mathrm{I}$ ' is calculated as:

$$
\operatorname{HM}\left(Z, \varsigma^{*}\right)\left[\begin{array}{ll}
-\operatorname{Lxx}\left(\mathrm{z}, \varsigma^{*}\right) & \operatorname{Lxy}\left(\mathrm{z}, \varsigma^{*}\right) \\
\operatorname{Lxy}\left(\mathrm{z}, \varsigma^{*}\right) & \operatorname{Lyy}\left(\mathrm{z}, \varsigma^{*}\right)
\end{array}\right]
$$


The convolution of the Gaussian is denoted by Lxx (z, $\left.\varsigma^{*}\right)$.

Consider using the Box filter and integral images to improve the speed of SURF. Using integral pictures at a cheap computational cost The Box filter can be computed, and the size of the filter has no effect on the calculation.

B. Orientation Assignment: By employing the Haar wavelet, it is possible to assign orientation to observed key points. Using a group of pixels, the Haar wavelet responses are computed in both directions around the detected spot, there is a 6 radius circles. As a result, summing the responses of the Haar wavelet determines the dominant orientation. It's possible to get local orientation by adding up all of the data from each location's $\mathrm{x}$ and $\mathrm{y}$ responses within a size of $\pi / 3$ orientation window. It is possible to establish the interest point orientation by looking at the longest vector among all of the windows.

C. Descriptors of SURF: unique description of a features specified by SURF descriptor. A description can be derived based on the area surrounding a point of interest in the data. It's possible to calculate a surf description using the responses of Haar wavelet as well as integral photos. To begin, create a square rectangle around the key point. An area of $4 * 4$ squares is divided into a wavelet-based sub-region of $20 \mathrm{~S}$. This technique keeps important spatial information, such as object locations. To give some robustness when deformations and translations occur, the interest areas are weighted using a Gaussian centered at the interest point. Each key point's Haar wavelet response at $5 S^{*} 5 S$ should be determined. A descriptor vector for the SURF algorithm can be written as $64 \mathrm{D}$ $\left(4^{*} 4^{*} 4=64\right)$. It's a lighting invariant SURF descriptor As a result of Haar; the reaction is invariant to lightning.

D. Matching Interest Points: After the preceding procedure's detection of robust key points, the following step is to match those interest points. The
Euclidean distance between the feature vectors determines whether or not these key points may be matched together. You may use it to find distance between the key points of a picture and its training image, and then use that distance to match up the key points.

\section{Hybrid approaches:}

Hybrid approaches, which use both local feature based and holistic methods at the same time, are the third type of traditional face recognition approaches. A popular research trend is to hybridize or combine multiple methodologies to achieve better outcomes. Many researchers use hybrid models to combine the benefits of local and global methodologies.

A hybrid method is proposed in ref [59] based on Principle Component Analysis with TRS (Tolerance Rough Similarity). In this hybrid approach, from the face image by using PCA the feature matrix is extracted, and then the similarity index is extracted using TRS. On multiple datasets (OUR, YALE, and ORL), the results attained good accuracy rates of up to ninety seven percent and showed consistency. Ref [60] takes a different strategy when it comes to facial recognition. Eigen-values of a 2Dim wavelet transform are utilized as k-means, correlation coefficient as preprocessing methods, and an RBFN network as a classifier to create a feature vector. Using the specified feature vectors, the minimum Euclidean distance for each person is determined. For a new face image, the feature vector is created first, and the distance between this new vector and all centres is compared to get the similarity index. Recognition accuracy was 96 percent in the best-case scenario.

\section{PCA with Tolerance Rough Similarity:}

Using PCA the relevant features from the query image are first obtained. After that An image is compared to images in the database using tolerance rough set-based similarity. The tolerance similarity 
measure was used to calculate approximation-based similarity values that were lower and greater.

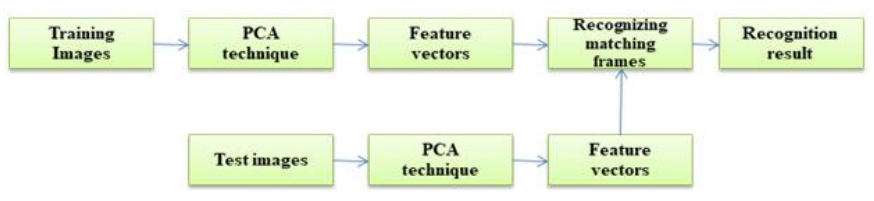

In general, pixels are used to represent each facial image. For example, the extracted features might be utilized directly for face recognition or further processed to choose relevant ones or reduce the quantity. In recent years.

A number of PCA-based methods have been presented. For the Characterization of Human Faces, a transformation termed Eigen space projection can be used [61]. PCA [62] stands for "passive face recognition". It is feasible to minimize the complexity of the image groupings by using PCA. Some of PCA's uses include computer access control, medical records, online banking, post office, passport verification, and etc.etc.

PCA uses following steps

1. Extract images of faces

Let us take a set of $\mathrm{K}$ face images $\left(\mathrm{I}_{1}, \mathrm{I}_{2} \ldots \ldots \ldots . . \mathrm{I}_{\mathrm{K}}\right)$ with size $n^{*} n$ and average of training set image

( $\mu) \frac{1}{K} \sum_{I=1}^{K} I i$

2. Subtract the mean value

$$
\mathrm{X}_{\mathrm{i}}=\mathrm{I}_{\mathrm{i}}-\mu
$$

3. Calculate the covariance

Covariance $=\mathrm{AA}^{\mathrm{T}}$

Where $\mathrm{A}=[\mathrm{X} 1, \mathrm{X} 2 \ldots \mathrm{XK}]$

4. Eigenvector (EV) and Eigen values are calculated as follows $(\lambda)$

$\mathrm{XEV}=\lambda \mathrm{EV}$

Where $\lambda$ is Eigen value and a set of eigenvector associated with it are represented by EV
5. form the feature vector and select the components

$\mathrm{FeV}=[\mathrm{EV} 1, \mathrm{EV} 2 \ldots \mathrm{EVn}]$

6. Transformed vector calculated by

Transformed data $=\mathrm{FeV}^{\mathrm{T}}(\mathrm{I}-\mu)$

Features are derived from facial photos using the algorithm above. Tolerance similarity is evaluated between pictures in the proposed work, in addition to distance (e.g., Euclidean distance).

The study of intelligent systems is known as rough set theory, this was expressed in Ref $[63,64]$. Rough set theory [65] is an excellent method for determining face recognition's most important features.

Rough set theory is a statistical technique used in multi-attribute decision analysis to analyze uncertainty and vagueness. There is a discussion of approximate set-based categorization algorithms in $[66,67]$. DS is for finite decision classes of a nonempty set. Let $R=(V, F A \cup S)$ be a decision table where $\mathrm{V}$ stands for finite elements of a non-empty set and FA stands for finite attributes of non-empty set. $\mathrm{V}=\mathrm{Ua}$, where $\mathrm{Ua}$ represents the collection of values of the attribute 'a'. For any $\mathrm{I} \subseteq$ FA There is an indiscernibility relation Indis(I) for any IFA, which can be described as

$$
\operatorname{Indis}(\mathrm{I})=\left\{\left(\mathrm{Zi}_{\left.\mathrm{i}, \mathrm{Z}_{\mathrm{j}}\right)} \in \mathrm{V}^{2} \quad \mid \quad \mathrm{f}_{\mathrm{i}}\right.\right.
$$

\section{(a) $\left.=\mathrm{f}_{\mathrm{j}}(\mathrm{a}), \forall \mathrm{a} \in \mathrm{FA}\right\}$}

In situations when $x i$ and $x j$ are indistinguishable, $\left(\mathrm{Zi}, \mathrm{Zj}_{\mathrm{j}}\right) \in \operatorname{Indis}(\mathrm{I})$. There are two types of classes that are generated by the Indis programme: Indis (I) For example, the element $\mathrm{Z}$ and the element V/S share the same class label, therefore $\mathrm{Z} \in \mathrm{V} / \mathrm{DS}$.

Algorithm:

INPUT: all face images I and their features $t$ and query face image

OUTPUT: Matched image

1. Extract the features from face image using PCA 
2. Check whether the extracted feature is uncertain

$$
\mathrm{X}\left(\mathrm{I}_{\mathrm{i}}\right)=\left\{\mathrm{I}_{\mathrm{j}} \mid \mathrm{F}\left(\mathrm{I}_{\mathrm{i}} \mathrm{I}_{\mathrm{j}}\right)>=2\right\} \mathrm{U}\left\{\mathrm{I}_{\mathrm{i}}\right\}
$$

3. Compute tolerance similarity to compare test image and trained image

$$
\mathrm{S}_{\mathrm{a}}\left(\mathrm{I}_{\mathrm{i}} \mathrm{I}_{\mathrm{j}}\right)=\mathrm{I}-\frac{\mid d(a(I i), a(I j) \mid}{|d \max |}
$$

4. Compute lower and upper approximation based on similarity

$$
\begin{aligned}
& \underline{t}(\mathrm{a}) \mathrm{I}=\left\{\mathrm{I}_{\mathrm{i}} \mid \mathrm{I}_{\mathrm{i}} \in \mathrm{U}, \mathrm{Sa}(\mathrm{I})=1\right\} \\
& \mathrm{t}(\mathrm{a}) \mathrm{I}=\left\{\mathrm{I}_{\mathrm{i}} \mid \mathrm{I}_{\mathrm{i}} \in \mathrm{U}, \mathrm{Sa}(\mathrm{I})>0\right\}
\end{aligned}
$$

5. Match test image with lower approximation if not match test image with upper approximation.

6. Output the matching result

\section{2) 2D wavelet transforms:}

A face recognition system that uses "Radial Basis Function" RBF neural network as a classifier and "wavelet transform" Eigen values as feature vectors is said to be 2-Dimensional wavelet transformation used to breakdown the face images. The wavelet coefficients are averaged from the wavelet transformation in order to determine centers of features. As part of the train process, four wavelet transform outputs are evaluated, and all of the Eigen values of these This is followed by storing as features the wavelet sub images with a maximum of 10 Eigen values Using wavelet transform with four sub images and 10 Eigen values for each sub image, the feature vector has a length of 40 elements.RBF function selects a centre for each individual based on the least Euclidean distance from all features after features have been obtained. When presented with a new face image, An RBF network computes a feature vector first, then compares the distance (error) of that new vector to the centers of all people to discover the shortest distance to aim at the target face.

Steps followed by 2-D wavelet transformation are

\section{Step 1: Preprocessing in wavelet transform:}

A new time domain coordinate system is created via the wavelet transformation. As a first-level in fig: 4, splitter, this transform splits the image into 4 new sub-images these are mapped into matrices, These new matrices have a lot of information about the image and are very resistant to noise and There are detailed coefficients of face that are decomposed in wavelet transformation as seen in the below fig .

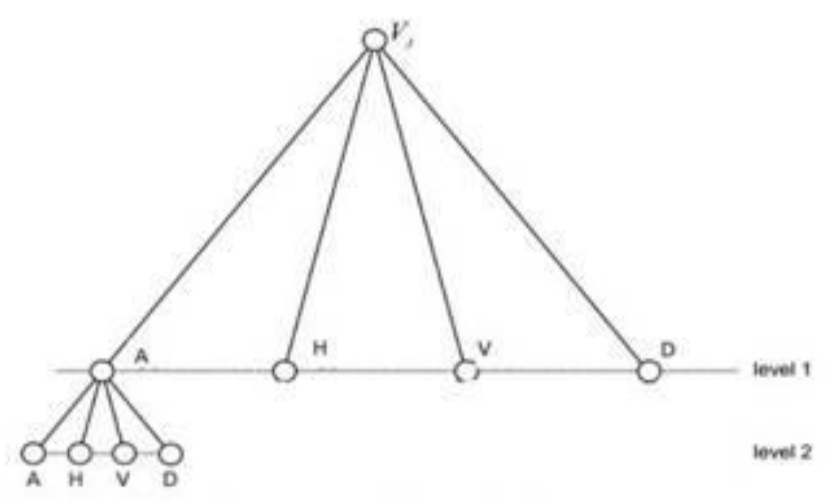

Fig 4: 2-D wavelet transformation

A scaled representation of the original image with all details, an approximated horizontal gradient, an approximated vertical gradient, and diagonal details can be used to break down the face picture $\mathrm{V}$ into up to four new images. If the algorithm requires more details, this step can be repeated until the size of the scaled image stored in A is smaller than a certain threshold. Similar to how Level 2 of the wavelet transformation decomposes only the A into approximation and details, a similar approach is used to break down horizontal, vertical, and diagonal details to create new sub images. Only two levels of face images were processed using the wavelet packet transformation. There are four matrices that arise from the original image being scaled down: two horizontal details; two vertical details; and two diagonal features from the original. It is used as recognition input to extract trustworthy features from the wavelet packets obtained during the preprocessing step.

\section{Step 2: Feature extraction:}

It is initially necessary to extract all wavelet sub matrix Eigen values and Eigen vectors in the feature extraction process. This is done as part of the preprocessing procedure, when we extract seven sub 
matrixes each sub matrix's Eigen values and eigenvectors have been calculated. In mathematics, eigenvectors are vectors with non-zero values proportional to the original vectors after being multiplied by a matrix.

$$
\mathrm{Mz}=\lambda \mathrm{z}
$$

$\mathrm{M}=$ Matrix Wavelet Sub Image $; \mathrm{z}=$ Eigenvector; $\lambda=$ Eigen value

A normalized Eigenvector is one of the most common methods of pattern detection. So, the Eigen values are the lines that pass through a matrix but are not transformed. That is obvious. That the lines that pass through the original matrix are known as Eigen values, But aren't altered in any way by the transformation. Identifying a matrix Eigen values is a pre-requisite to obtaining the Eigenvectors of that matrix. If you want to discover Eigen values, you'll need to know what the M-determiner of $M-\lambda I$ is equal to. It is possible to find Eigenvectors by replacing our Eigen values into our original equation after we have a set of Eigen values.

When all Eigen values have been found, the values are sorted, and the ten highest values are selected it's a fact, the entire number of elements of the matrix is summarized in 10 higher Face picture is separated into seven sub matrix using level 2's wavelet transform in the preparation step. A vector of length $7 * 10=70$ can be obtained by selecting 10 Eigen values from each sub matrix.

As part of the train process, certain facial photos of each individual are taken from a variety of angles and viewpoints. After a preprocessing phase, The Eigen values of wavelet sub matrixes from these images are extracted.

Correlation coefficients are obtained after detecting the features of a person face image, and if a vector or feature is so dissimilar from other vectors or features, it is discarded during the train phase. It is also possible to improve the performance of features by using k-means clustering method.

\section{Step 3: Noise reduction by K-means clustering} method.

The K-means clustering algorithm, which has its origins in signal processing, is frequently used for cluster analysis, both in image processing and data mining. This approach maps $\mathrm{N}$ observations or feature vectors into one or more $\mathrm{k}$ clusters or centres using $\mathrm{K}$ means clustering.

Despite the fact that this problem is challenging computationally, there are several effective heuristic techniques that can discover centers' very rapidly however, k-means clustering attempts to discover groups of identical components, whereas the expectation-maximization technique allows for varied geometries of clusters. A centre diverge occurs when features are sparse or so distinct from one another that algorithms aren't capable of grouping them all together in defined centers'. The k-means algorithm diverges when a vector or feature causes it to do so. This feature should be removed in the train stage.

\section{Modern Approaches:}

Face Recognition and Fuzzy Logic: As previously mentioned, major difficult issues in face recognition systems is dealing with variations in illumination. A wide range of approaches have been offered over the last few decades, but no solution has been totally successful in dealing with illumination variance. In this case, fuzzy set theory has been shown to produce excellent outcomes.

[131] is a fuzzy-based facial recognition model from the early 1990s. For a class that was regarded a membership grade with prediction, the proposed models used an incremental degree of projection. This level of discrimination helps with categorization accuracy. When working with the features vectors generated by PCA, a fuzzy K-nearest neighbour class assignment is used. When the results of these studies are compared to the Yale, ORL, and CNU (Chungbuk National University) face databases, it is clear that the categorization results have improved dramatically. Since the beginning of the profession, face 
recognition researchers have struggled with illumination variations.

\section{In Fuzzy based Face Recognition Recent Developments:}

2DPCA a fuzzy-based face recognition algorithm has been presented in ref [143]. It uses the K-nearest neighbor fuzzy approach and creates the training samples membership degree matrix, which is then used to construct fuzzy means for each class. A prediction can be made using these fuzzy means in order to improve classification results. Face datasets from FERET, ORL, and YALE are used to test the proposed model. Experiments show that the method works effectively even in difficult environments including lighting expression variations and position limitations.

Face recognition researchers have struggled with illumination variations from the beginning of the field. [70] Developed a fuzzy reasoning model to deal with light variations using a fuzzy theory-based picture enhancement strategy, it's an adaptive development method that accentuates low contrasts and non-uniform illumination. The proposed approach, Increases the intensity and dissimilarity of the original image. It also brings out the hidden aspects of the provided image because to its fuzzy logic.

Four blind reference image quality measures derived from the input visual face were used to evaluate the fuzzy reasoning model. The information from these four matrices (X, Y, Z, and $\mathrm{M}$ ) is then used to restructure an augmented picture using a fuzzy logic system. The authors also compare six state-of-the-art approaches to the proposed model in a comparative analysis. It achieves impressive results for position, illumination, and expression on well-known face datasets "Yale-B, Mobio, FERET, and Carnegie Mellon University". Other research, In ref [71] and ref [72], have incorporated fuzzy linear regression discriminant projection models in a similar direction.

\section{2)Machine learning:}

A pioneer in computer games and artificial intelligence, Arthur Samuel developed the phrase Machine Learning in 1959, stating that it "gives computers the ability to learn without being explicitly taught." "A computer programme is said to learn from experience $\mathrm{E}$ with respect to some task $\mathrm{T}$ and some performance measure $\mathrm{P}$, if its performance on $\mathrm{T}$, as measured by $\mathrm{P}$, increases with experience $\mathrm{E}$," remarked in 1997 by Tom Mitchell in a mathematical and relational definition.

As a result, machine-learning implementations categorized into three broad types, which are as follows: -

\section{Supervised learning:}

In this technique well-labeled data used to train the machines, they may predict the output based on that data. For instance, there are inputs which have already been labeled with the desired result. Machines are taught how to predict output accurately through supervised learning, which relies on training data provided by humans. A student learns the same notion under the guidance of a teacher. When you supervise machine learning, you provide the model with both input data and the proper output data.

\section{Un Supervised learning:}

This type of machine learning analyses and clusters unlabeled datasets using machine learning algorithms, often recognized as unsupervised machine learning.

\section{Reinforcement learning:}

An agent learns to behave in an environment by executing actions and seeing the effects. The agent receives positive feedback for every good action, and negative feedback or a penalty for every bad action. For example, with Reinforcement Learning, instead of labeled data, the agent is learned by using feedback. We don't have any pre-labeled data, so the agent can only learn from its own experience. A certain sort of problem can be solved with RL, such as game-playing, robotics, etc., where decision making is sequential and the aim is long-term. The agent has a relationship with the environment.

Machine learning in face recognition: 
"Artificial Intelligence (AI), Machine Learning (ML), and Deep Learning (DL)" technologies are allowing the facial recognition Using facial recognition technology, a person's identity can be determined by analyzing his or her face. Algorithms for machine learning are used to identify face features and match them to photographs of people in a database.

It is difficult to understand how facial recognition technology works, which is why a thorough explanation would go much beyond the scope of this article A machine's ability to detect a face is based on four fundamental problems. Facial recognition and facial verification are among the techniques that can be used for face detection, face alignment

Face detection:

For the machine to detect the face in a picture or video, it must first locate the facial features in the Face detection is now a standard feature on most cameras. Facial recognition is also used by social media sites such as Snap Chat and Face book to allow users to contribute.

Face alignment:

Unaligned faces appear completely different on a computer. There is a need for an algorithm to normalize a face in order for it to be consistent Using a variety of generic face landmarks is one approach to achieve this When it comes to your chin, your nose's tip and even your eyes' outer edges - as well as other areas around your eyes and mouth Now we need to train an algorithm that can locate these places and turn the faces towards the centre.

Feature measurement and extraction:

In this step, numerous facial traits are measured and extracted in order for the algorithm to match the face with other faces in its database. After much confusion about which features should be measured and extracted, researchers realized it would be better if they just left it up the ML algorithm to decide what measurements to take on its own Embedding employs deep convolutional neural networks to teach itself to generate many measurements of a face, allowing it to identify it from other faces in a crowd.
Face Recognition: A final ML algorithm will compare the measures of the face to known faces in a database using the unique measurements of each face. Whatever face in your database most closely resembles your subject's face in terms of its measurements will be returned as the match.

\section{Face verification:}

It compares the distinctive qualities of one face to those of another. To determine if the faces match, the ML algorithm returns a confidence value.

\section{Dictionary learning:}

This is a subset of machine learning technique, The fundamental goal of dictionary learning for face recognition is to generate a dictionary matrix from a sparse representation of training data. The discriminative features are retrieved from the training data in this case by learning the required vocabulary. Assuming we have a random distribution of face samples. The success of the sparse representation is dependent on the learned dictionary [83]. We must construct a task-specific vocabulary using the available face photographs. As a result, existing feature representation theories and approaches must be recreated for dictionary learning as a new research topic.

Ref [84] suggested a face recognition framework for video streaming based on extended dictionary learning. The approach has been shown to be resistant to changes in lighting, position, and video sequence modifications. For the first phase, input videos are partitioned into a group of frames with the same illumination effect, and they are all invariant with respect to their pose. To prevent temporal repetition, this group-based framing method considers alternating positions and illumination. As a result of sparse representation, representation error is reduced for each collection of frames.

In a second step, these learnt dictionaries are concatenated to generate a global dictionary that is sequence-specific in structure. After that, the third phase is used only for recognition. As a result of this prediction, automatic recognition becomes more cost- 
effective. On three of the most difficult datasets for video-based face recognition ("the Face and Ocular Challenge Series (FOCS), the Multiple Biometric Grand Challenge (MBGC), and the Honda/UCSD datasets"), there is a significant improvement in performance when compared to earlier methods.

3) Face recognition based on Deep learning methods:

Deep neural networks have been discovered to have huge processing capability for object recognition; As a result, deep neural networks have transformed machine learning in the past several years. From the social sciences to engineering to biological sciences and beyond, researchers are looking at deep frameworks in order to combine their existing models and come up with novel results It has a outstanding processing authority, great accuracy, and resultoriented behavior, according to several researchers, including those in the face recognition field $[73,74]$. This section provides a concise overview of recent deep learning-based achievements in facial recognition.

The use of facial recognition to enhance accuracy, deep learning mostly depends on integrating current models with deep networks. Improved facial recognition systems are being developed. Deep neural networks and similar technologies are well suited to high accuracy and robustness in terms of performance. It has an advantage over typical facial recognition algorithms due to its superior ability to reliably categorize a large number of unlabeled faces. Working with unconstrained deep models in a dynamically changeable face environment like position, illumination, and cosmetic effects has shown some impressive results. Deep learning techniques for face recognition have been evaluated; however there is still a big gap between them against numerous variations.

Ref $[75,76]$ explore the use of ANN for large-scale feature representation in early applications of face recognition. Final result, hidden layers are maximized in neural networks. According to this theory, To extract features space in a generic approach, There are multiple layers in the deep model, each one constructed on a kernel function that has a particular optimization target [77]. Unbelievable for identifying purposes because it is able to automatically without any human participation learn from unlabeled data. As a result of the usage of deep belief networks in 2006 [78], the hybridization of multiple techniques by Sun et al. $[79,80,81]$ and the categorization of Image Net with CNN [82], face recognition research has entered a new era.

\section{Conclusion}

The term "Face recognition research" has become extremely popular research areas in the fields of "machine learning, artificial intelligence, and pattern recognition". It has a huge impact on the developing digital society because it has so many social and scientific uses. We investigated a variety of traditional appearance-based techniques as well as a number of recent machine learning-based approaches for face recognition such as deep learning, and dictionary learning in this study.

On numerous data sets such as "MS-Celeb-1M, YALE, AT\&T, AR, 3DMAD, GEORGIA, Pub Fig, and CMUPIE", the accuracy attained by different researchers has been examined. Reduced computational costs, illumination variations, viewpoint tolerance, scale and rotation variations, and the collection of 3D data have all been explored. There are many unsolved questions in future occlusion and background subtraction research. Finally, after assessing several methodologies, we suggest that incorporating soft biometrics with this exciting research area, other existing facial recognition algorithms can produce extraordinary results.

\section{REFERENCES}

[1]. Poon G, Kwan KC, Pang W (2019) Occlusionrobust bimanual gesture recognition by fusing 
M. ShalimaSulthana et al Int. J. Sci. Res. Comput. Sci. Eng. Inf. Technol, November-December-2021, 7 (6) : 57-79

multi-views. Multimed Tools Appl 78 (16):23469-23488

[2]. Wong Y, Chen S, Mau S, Sanderson C, Lovell BC (2011) Patch-based probabilistic image quality assessment for face selection and improved video-based face recognition. In: IEEE Biometrics workshop, computer vision and pattern recognition (CVPR) workshops. IEEE, pp 81-88

[3]. Ouyang D, Zhang Y, Shao J (2019) Video-based person re-identification via spatio-temporal attentional and two-stream fusion convolutional networks. Pattern Recogn Lett 117:153-160.

[4]. VS Manjula, Lt Dr S Santhosh Baboo, et al. Face detection identification

[5]. and tracking by prdit algorithm using image database for crime investigation. International JournalofComputerApplications,38(10):4046,2012.

[6]. Karen Lander, Vicki Bruce, and Markus Bindemann. Use-inspired basic research on individual differences in face identification: Implications for criminal investigation and security. Cognitive research: principles and implications,3(1):1-13,2018.

[7]. "Yongmei $\mathrm{Hu}$, Heng An, Yubing Guo, Chunxiao Zhang, and Ye Li. The development status and prospects on the face recognition. In Bioinformatics and Biomedical Engineering (iCBBE), 2010 4th International Conference on, 2010."

[8]. Garain J, Kumar RK, Kisku DR, Sanyal G (2019) Addressing facial dynamics using $\mathrm{k}$-medoids cohort selection algorithm for face recognition. Multimed Tools Appl 78(13):18443-18474.

[9]. Mahmood A, Uzair M, Al-máadeed S (2018) Multi-order statistical descriptors for real-time face recognition and object classification. IEEE Access 6:12993-13004.

[10]. Naoufel Werghi, Claudio Tortorici, Stefano Berretti and Alberto Del Bimbo, "Boosting 3D
LBP-Based Face Recognition by Fusing Shape and Texture Descriptors on the Mesh", IEEE Transactions on Information Forensics and Security, Vol. 11, Issue 5, pp. 964 - 979, Jan 2016

[11]. Jiwen Lu , Venice Erin Liong and Jie Zhou, "Simultaneous Local Binary Feature Learning and Encoding for Homogeneous and Heterogeneous Face Recognition", IEEE Transactions on Pattern Analysis and Machine Intelligence, Vol. PP, Issue 99, pp.1-1, Aug 2017.

[12]. Swati Manhotra and Reecha Sharma, "Facial Feature Extraction Using Local Binary Pattern and Local Ternary Pattern with Gradient Based Illumination Normalization", Internaltional Journal of Advanced Research in Computer Science, Vol. 8, Issue 7, Aug 2017.

[13]. Rajesh Kumar Gupta,Umesh Kumar Sahu ," Real Time Face Recognition under Different Conditions “,International Journal of Advanced Research in Computer Science and Software Engineering Volume 3, Issue 1, January 2013 ,86-89.

[14]. C.Nagaraju, B.Srinu, E. Srinivasa Rao- An efficient Facial Features extraction Technique for Face Recognition system Using Local Binary Patterns\| International Journal of Innovative Technology and Exploring Engineering (IJITEE) ISSN: 2278 -3075, Volume-2, Issue-6, May 2013.

[15]. Di Huang, Caifeng Shan, Mohsen Ardabilian, Yunhong Wang and Liming Chen-Local Binary Patterns and Its Application to Facial Image Analysis: A Survey-IEEE Transactions On Systems, man, and cybernetics-part c: applications and reviews, vol. 41, no. 6, november 2011.

[16]. Huang, D.; Shan, C.; Ardabilian, M.; Wang, Y.; Chen, L. Local binary patterns and its application to facial image analysis: A survey. 
IEEE Trans. Syst. Man Cybern. Part C Appl. Rev. 2011, 41, 765-781. 13.

[17]. Belhumeur, P.N.; Hespanha, J.P.; Kriegman, D. Eigenfaces vs. fisherfaces: Recognition using class specific linear projection. IEEE Trans. Pattern Anal. Mach. Intell. 1997, 19, 711-720.

[18]. Liu, C.; Wechsler, H. Gabor feature based classification using the enhanced fisher linear discriminant model for face recognition. IEEE Trans. Image Process. 2002, 11, 467-476.

[19]. Lowe, D.G. Distinctive image features from scale-invariant key points. Int. J. Comput. Vision 2004, 60, 91-110.

[20]. Zhang, J.; Yan, Y.; Lades, M. Face recognition: Eigen face, elastic matching, and neural nets. Proc. IEEE 1997, 85, 1423-1435.

[21]. Wright, J.; Yang, A.Y.; Ganesh, A.; Sastry, S.S.; $\mathrm{Ma}, \mathrm{Y}$. Robust face recognition via sparse representation. IEEE Trans. Pattern Anal. Mach. Intell. 2009, 31, 210-227.

[22]. Y. Pang, Y. Yuan and X. Li, Iterative subspace analysis based on feature line distance, Image Processing, IEEE Transactions on, vol.18, pp.903-907, 2009.

[23]. S. Lawrence, C. Giles, A. C. Tsoi and A. Back, Face recognition: A convolutional neuralnetwork approach, Neural Networks, IEEE Transactions on, vol.8, pp.98-113, 1997.

[24]. S. Yan, H. Wang, X. Tang and T. Huang, Exploring feature descriptors for face recognition, Acoustics, Speech and Signal Processing, IEEE International Conference on, vol.1, pp.629-632, 2007.

[25]. Q. Z. C. Zhou, X. Wei and B. Xiao, Image reconstruction for face recognition based on fast ICA , International Journal of Innovative Computing, Information and Control, vol.4, no.7, pp.1723-1732, 2008.

[26]. J. Yang, A. Frangi, J.-Y. Yang, D. Zhang and Z. Jin, Kpca plus lda: A complete kernel fisher discriminant framework for feature extraction and recognition, IEEE Transactions on Pattern
Analysis and Machine Intelligence, vol.27, pp.230-244, 2005.

[27]. C. Naga Raju, SivaPriya. T, prudvi.ch "A novel method for recognizing face to indicate the state of emotion in order to avoid consistent effect on decisions making" has been published in International Journal of Advancements in Computer Science \& Information Technology (IJACSIT) September 2011Edition.pp.10-17.

[28]. Kanade T (1973) Picture processing system by computer complex and recognition of human faces. Ph.D. thesis, Kyoto University, Japan.

[29]. Sirovich L, Kirby M (1987) Low-dimensional procedure for the characterization of human faces. J Opt Soc Am A 4(3):519-524

[30]. Turk M, Pentland A (1991) Eigenfaces for recognition. J Cogn Neurosci 3(1):71-86

[31]. Etemad K, Chellappa R (1997) Discriminant analysis for recognition of human face images. In: International conference on audio- and video-based biometric person authentication, pp 125-142.

[32]. Guo G, Li S, Chan KL (2000) Face recognition by support vector machines. In: 4Th IEEE international conference on automatic face and gesture recognition (FG 2000), Grenoble, pp 196-201

[33]. Zhao W, Chellappa R, Phillips PJ (1999) Subspace linear discriminant analysis for face recognition. Citeseer

[34]. Bartlett MS, Movellan JR, Sejnowski TJ (2002) Face recognition by independent component analysis. IEEE Trans Neural Netw 13(6):14501464.

[35]. Rajkiran Gottumukkal and Vijayan K Asari. An improved face recognition technique based on modular pca approach. Pattern Recognition Letters, 25(4):429-436, 2004.

[36]. D., C., Hoyle, M., and Rattray. Pca learning for sparse high-dimensional data. Epl, 2003.

[37]. K. Vijay and K. Selvakumar. Brain fmri clustering using interaction k-means algorithm 
with pca. In 2015 International Conference on Communications and Signal Processing (ICCSP), 2015

[38]. Jianke Li, Baojun Zhao, Zhang Hui, and Jichao Jiao. Face recognition system using svm classifier and feature extraction by pca and lda combination. In Computational Intelligence and Software Engineering, 2009.

[39]. CiSE 2009. International Conference on, 2010.

[40]. Frank Vogt, Boris Mizaikoff, and Maurus Tacke. Numerical methods for accelerating the pca of large data sets applied to hyperspectral imaging. InEnvironmental \& Industrial Sensing, 2002.

[41]. Carlos Ordonez, Naveen Mohanam, and Carlos Garcia-Alvarado. Pca for large data sets with parallel data summarization. Distributed \& Parallel Databases, 32(3):377-403, 2014.

[42]. Shireesha Chintalapati and MV Raghunadh. Automated attendance management system based on face recognition algorithms. In 2013 IEEE International Conference on Computational Intelligence and Computing Research, pages 1-5. IEEE, 2013.

[43]. Juwei Lu, Kostantinos N. Plataniotis, and Anastasios N. Venetsanopoulos. Face recognition using lda-based algorithms. IEEE Transactions on Neural Networks, 14(1):195200, 2003.

[44]. Jianke Li, Baojun Zhao, Zhang Hui, and Jichao Jiao. Face recognition system using svm classifier and feature extraction by pca and lda combination. In Computational Intelligence and Software Engineering, 2009. CiSE 2009. International Conference on, 2010.

[45]. M. Turk and A. Pentland, "Eigenfaces for recognition," J. Cognitive Neurosci., vol. 3, no. 1, pp. 71-86, 1991.

[46]. G. Cottrell and J. Metcalfe, "Face, gender and emotion recognition using holons," in Advances in Neural Information Processing Systems, D. Touretzky, Ed. San Mateo, CA: Morgan Kaufmann, 1991, vol. 3, pp. 564-571
[47]. P. S. Penev and J. J. Atick, "Local feature analysis: A general statistical theory for object representation," Network: Comput. Neural Syst., vol. 7, no. 3, pp. 477-500, 1996

[48]. P. Comon, "Independent component analysisA new concept?," Signal Processing, vol. 36, pp. 287-314, 1994

[49]. A. Pentland, B. Moghaddam, and T. Starner, "View-based and modular eigenspaces for face recognition," in Proc. IEEE Conf. Comput. Vision Pattern Recognition, 1994, pp. 84-91.

[50]. M. Turk and A. Pentland, "Eigenfaces for recognition," J. Cognitive Neurosci., vol. 3, no. 1, pp. 71-86, 1991.

[51]. A. J. Bell and T. J. Sejnowski, "An informationmaximization approach to blind separation and blind deconvolution," Neural Comput., vol. 7, no. 6, pp. 1129-1159, 1995.

[52]. A. Cichocki, R. Unbehauen, and E. Rummert, "Robust learning algorithm for blind separation of signals," Electron. Lett., vol. 30, no. 7, pp. 1386-1387, 1994.

[53]. P. Comon, "Independent component analysisA new concept?," Signal Processing, vol. 36, pp. 287-314, 1994.

[54]. S. Laughlin, "A simple coding procedure enhances a neuron's information capacity," Z. Naturforsch., vol. 36, pp. 910-912, 1981.

[55]. Cortescorinna and Vapnikvladimir. Supportvector networks. Machine Learning, 1995.

[56]. Aixin Sun, Ee-Peng Lim, and Ying Liu. On strategies for imbalanced text classification using svm: A comparative study. Decision Support Systems, 48(1):191-201, 2009.

[57]. Ahonen T, Hadid A, Pietikàinen M (2004) Face recognition with local binary patterns. In: Proceedings of 8th European Conference on Computer Vision-ECCV, Prague, Czech Republic, Part I, pp 469-481.

[58]. Tan X, Triggs B (2010) Enhanced local texture feature sets for face recognition under difficult 
M. ShalimaSulthana et al Int. J. Sci. Res. Comput. Sci. Eng. Inf. Technol, November-December-2021, 7 (6) : 57-79

lighting conditions. IEEE Trans Image Process 19(6):1635-1650Return to ref 239 in article.

[59]. J Krizaj, V. Struc, N Pavesi, "Adaptation of SIFT Features for Face Recognition under Varying Illumination", MIPRO 2010, May 24-28, 2010, Opatija, Croatia.

[60]. Haeseong Lee1, Semi Jeon, Inhye Yoon, and Joonki Paik1, "Recent Advances in Feature Detectors and Descriptors: A Survey", IEIE Transactions on Smart Processing and Computing, vol. 5, no. 3, June 2016http://dx.doi.org/10.5573/IEIESPC.2016.5.3 .153.

[61]. Lavanya B, Inbarani HH (2018) A novel hybrid approach based on principal component analysis and tolerance rough similarity for face identification. Neural Comput Appl 29(8):289299.

[62]. Hashemi VH, Gharahbagh AA (2015) Article:a novel hybrid method for face recognition based on $2 \mathrm{~d}$ wavelet and singular value decomposition. Amer J Netw Commun 4(4):9094.

[63]. Kirby M, Sirovich L (1990) Application of the KarhumenLoeve procedure for the characterization of human faces. IEEE Transactions on Pattern Analysis Machine Intelligence 12(1):103-108

[64]. Solunke V, Kudle P, Bhise A, Naik A, Prasad JR (2014) A comparison between feature extraction techniques for face recognition. International Journal of Emerging Research in Management \& Technology 3:38-41.

[65]. Pawlak Z (1982) Rough sets. Int J Parallel Prog 11(5):341-356 Pawlak Z (2002) Rough set theory and its applications. Journal of Telecommunications and information technology:7-10.

[66]. Swiniarski, R. (2000). An application of rough sets and Haar wavelets to face recognition. In International Conference on Rough Sets and
Current Trends in Computing. Springer Berlin Heidelberg. pp. 561-568.

[67]. Kim D (2001) Data classification based on tolerant rough set. Pattern Recogn 34(8):16131624

[68]. Kim D, Bang SY (2000) A handwritten numeral character classification using tolerant rough set. IEEE Trans Pattern Anal Mach Intell 22(9):923-937.

[69]. Kwak KC, Pedrycz W (2005) Face recognition using a fuzzy fisherface classifier. Pattern Recogn 38(10):1717-1732.

[70]. Li X (2014) Face recognition method based on fuzzy 2dpca. J Electr Comput Eng 2014:919041:1-919041:7

[71]. Oulefki A, Mustapha A, Boutellaa E, Bengherabi M, Tifarine AA (2018) Fuzzy reasoning model to improve face illumination invariance. SIViP 12 (3):421-428

[72]. Huang P, Gao G, Qian C, Yang G, Yang Z (2017) Fuzzy linear regression discriminant projection for face recognition. IEEE Access 5:4340-4349

[73]. Campomanes-Alvarez C, Älvarez BRC, Guadarrama S, Ibảnez Ȯ, Cordón O (2017) An experimental study on fuzzy distances for skullface overlay in craniofacial superimposition. Fuzzy Sets Syst 318:100-119

[74]. Arashloo SR (2016) A comparison of deep multilayer networks and markov random field matching models for face recognition in the wild. IET Comput Vis 10(6):466-474.

[75]. Sun Y, Wang X, Tang X (2013) Hybrid deep learning for face verification. In: IEEE International conference on computer vision, ICCV, Sydney, pp 1489-1496.

[76]. Stonham TJ (1986) Practical Face Recognition and Verification with Wisard. Springer, Netherlands, pp 426-441Return to ref 230 in article

[77]. Zhao W, Chellappa R, Phillips PJ, Rosenfeld A (2003) Face recognition: a literature survey. 
M. ShalimaSulthana et al Int. J. Sci. Res. Comput. Sci. Eng. Inf. Technol, November-December-2021, 7 (6) : 57-79

ACM Comput Surv (CSUR) 35(4):399458Return to ref 298 in article

[78]. Arashloo SR (2016) A comparison of deep multilayer networks and markov random field matching models for face recognition in the wild. IET Comput Vis 10(6):466-474Return to ref 15 in article

[79]. Hinton GE, Osindero S, Teh YW (2006) A fast learning algorithm for deep belief nets. Neural Comput 18(7):1527-1554.

[80]. Sun Y, Wang X, Tang X (2014) Deep learning face representation from predicting 10, 000 classes. In: IEEE Conference on computer vision and pattern recognition, CVPR, Columbus, pp 1891-1898.

[81]. Sun Y, Chen Y, Wang X, Tang X (2014) Deep learning face representation by joint identification-verification. In: Proceedings of the 27th International Conference on Neural Information Processing Systems - Vo.lume 2, NIPS, pp 1988-1996

[82]. Sun $Y$, Liang D, Wang X, Tang X (2015) Deepid3: Face recognition with very deep neural networks. CoRR arXiv:1502.00873

[83]. Krizhevsky A, Sutskever I, Hinton GE (2017) Imagenet classification with deep convolutional neural networks. Commun ACM 60(6):84-90.

[84]. Chen Y, Patel VM, Phillips PJ, Chellappa R (2012) Dictionary-based face recognition from video. In: Proceedings of 12th European Conference on Computer Vision ECCV, Florence, Part VI, pp 766-779.

\section{Cite this article as :}

M. ShalimaSulthana, C. NagaRaju, "A Survey on Classical and Modern Face Recognition Techniques ", International Journal of Scientific Research in Computer Science, Engineering and Information Technology (IJSRCSEIT), ISSN : 2456-3307, Volume 7
Issue 6, pp. 57-79, November-December 2021. Available at

doi $\quad$ : https://doi.org/10.32628/CSEIT21762

Journal URL : https://ijsrcseit.com/CSEIT21762

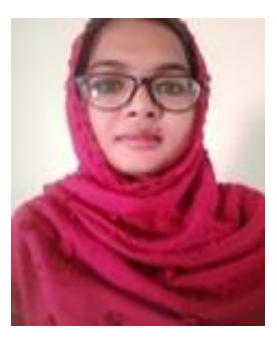

Mannuru Shalima Sulthana pursuing her $\mathrm{PhD}$ in the field of Machine learning under the supervision of C.NagaRaju Professor of Computer Science and Engineering, YSR Engineering College of Yogivemana University,Proddatur,kadapa,, Andhra Pradesh. She received her B.Tech in Information Technology at SSITS Rayachoty. M.Tech in Computer Science and Engineering at SSITS Rayachoty. She has six years of teaching experience as an Assistant Professor in the department of Computer and Engineering at IIIT RGUKT RKVALLEY Idupulapaya; she has published fifteen Research papers in various national and International journals and Conferences. She has attended twelve Faculty Development Programs and workshops. She is member of various professional societies like IAENG (International Association of Engineers).

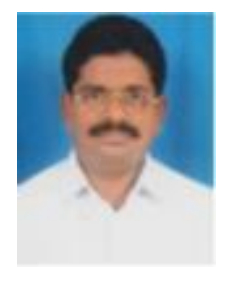

C. Naga Raju is currently working as professor in the Department of Computer Science and Engineering at YSR Engineering College of Yogi Vemana University, Proddatur, YSR Kadapa District, Andhra Pradesh, India. He received his B.Tech. In Computer Science and Engineering from J.N.T. University, Anantapur, and M.Tech. In Computer Science and Engineering from J.N.T. University Hyderabad and Ph.D. in Digital Image Processing from J.N.T. University Hyderabad. He has got 25 years of teaching experience. He received research excellence award, teaching excellence award, outstanding scientist award and Rayalaseema Vidhyaratna award for his credit. He wrote text books 
on programming in C, Network Security and Digital Image processing. He has got one patent on his research work. He has completed two DST funding projects worth of forty lakhs. Under his guidance seven $\mathrm{PhDs}$ are completed and five scholars are pursuing. He has published ninety-eight research papers in various National and International Journals and thirty research papers in various National and International Conferences. He has attended twenty seminars and workshops. He delivered more than seventy keynote addresses. He is member of various professional societies like IEEE, ISTE and CSI 